\title{
Difficult decisions
}

\author{
Conditional approval aims to speed the delivery of drugs to patients in need. But when full approval is denied, \\ complications arise for patients, companies and regulatory agencies alike.
}

n 2008, Avastin (Genentech) was conditionally approved for the treatment of metastatic breast cancer through the US Food and Drug Administration's (FDA) accelerated approval process. Approval was based on the results of the phase 3 E2100 trial, which showed that treatment with Avastin plus paclitaxel conferred a 5.5-month increase in median progression-free survival (PFS) over treatment with paclitaxel alone. In the conditional approval letter for Avastin, the FDA stated that follow-up trials were required to "further define the degree of clinical benefit to patients" for conversion to full approval.

But the next two phase 3 trials of Avastin in breast cancer patientsAVADO and RIBBON1 - showed more modest increases in median PFS (0.9-2.9 months). Moreover, a fourth trial, AVF2119g, of capecitabine with or without Avastin, showed no improvement in PFS in the Avastin arm. These results-coupled with what the FDA contends to be increased risks of serious side effects associated with Avastin in combination with standard chemotherapy and no increase in overall survival-led the agency to recommend in December 2010 that the approval of Avastin for breast cancer (but not for other indications) be withdrawn.

On June 29, following a two-day hearing requested by Genentech, the FDA oncologic drugs advisory committee voted unanimously against the continued approval of Avastin for the treatment of metastatic breast cancer. A final decision will be made by the FDA commissioner, Margaret Hamburg, some time after the close of the public comment period on July 28.

In a diametrically opposed move on June 30, the European Commission (EC) voted to broaden its approval of Avastin to include its combination with capecitabine as a frontline treatment option for metastatic breast cancer. And on the same day, the US Centers for Medicare and Medicaid (CMS) announced that they would continue to pay for Avastin to treat breast cancer, even if the FDA revokes its approval for this indication.

The firm statements made by these agencies belie the intricacies of their decisions. The FDA is well within its rights to deny full approval on the basis that the follow-up studies did not confirm the original increase in PFS reported in the E2100 trial. However, the language in the FDA's conditional approval letter (cited above) is vague. Moreover, according to Genentech, in an informal teleconference in 2009, the FDA indicated that full approval required "demonstrated improvement in PFS and evidence that survival is not impaired" (Avastin Briefing Book, 16 June 2010), leaving the extent of improvement necessary for approval open to interpretation.
Although the EC has now approved Avastin plus capecitabine for metastatic breast cancer, it did so after Genentech requested a review of an earlier decision against the use of Avastin with any standard cytotoxic chemotherapy-including capecitabine-as a first-line therapy for this indication because of its associated toxicity and the lack of measurable benefit to overall survival or quality of life. The EC reversed its original decision on the basis of data from the RIBBON1 trial, but only for a subset of breast cancer patients for whom other chemotherapies, including taxanes and anthracyclines, are not appropriate and who therefore have limited treatment options. Avastin plus paclitaxel is approved by the EC for the treatment of metastatic breast cancer and the change extends its authorized use. Accordingly, the EC and the FDA may have similar views of Avastin's efficacy in certain settings than might be inferred from their opposing decisions, but they appear to differ in their interpretation of what constitutes potential patient benefit.

It's possible that the FDA, too, might ultimately factor the paucity of treatment options for metastatic breast cancer into its final deliberations. But before a verdict, the CMS, a US government agency, should have deferred its announcement of the continued coverage of Avastin for breast cancer. Not only does the CMS statement appear to preempt the role of the FDA in regulating the approval of safe and efficacious drugs, it also has the potential to unduly influence the Commissioner's decision.

In particular, the scenario in which the CMS continues to pay for the treatment of an indication with a drug whose approval for that indication has been revoked by the FDA raises some pressing questions. Would the CMS be at fault or liable in the instance of adverse events in patients? And would the CMS help patients and physicians navigate treatment options that include a drug whose approval has been revoked? While the CMS has said that it will continue to evaluate its coverage of Avastin, making this statement would have sufficed, rather than expressing apparent disregard for the FDA's mandate to ensure patient health and safety.

In the end there are no clear winners here. Revoking Avastin's approval for breast cancer might deprive some patients of a potentially lifesaving treatment option, whereas granting full approval might expose some patients to excessive toxicity. But the studies conducted thus far have not identified the patients who may benefit most. The FDA needs to consider how this situation might have been avoided and whether its criteria for approval are well calibrated and well communicated at every step of the process. 